\section{Eggerthella lenta bacteremia in a Crohn's disease patient after ileocecal resection}

\author{
Venkata R Thota ${ }^{\dagger 1}$, Sunil Dacha', Aparna Natarajan' \& Judith Nerad' \\ 'Department of Medicine, Saint Joseph Hospital, 2900 N Lake Shore Dr, Chicago 60657, IL, USA \\ ${ }^{\dagger}$ Author for correspondence: drthota@gmail.com
}

Eggerthella lenta is an anaerobic, nonspore-forming Gram-positive rod and is a common gut commensal. Bacteremia from this organism is rare but when present is always clinically significant. Gastrointestinal disease and malignancy are the most common causes for bacteremia from this organism. Eggerthella species have been isolated in feces from patients with inflammatory bowel disease, but bacteremia has not been reported to the best of our knowledge. Here we report the case of a young African-American female with Crohn's disease who developed Eggerthella lenta bacteremia after ileocaecal resection.

Anaerobic Gram-positive bacilli such as Clostridium, Propionibacterium, Bifidobacterium, Eubacterium, Lactobacillus, Eggerthella are common gut commensals [1]. Although bacteremia associated with them is more commonly contaminant, it can be clinically significant, indicating underlying active intestinal process. It has also been reported that isolation of Eggerthella from blood is almost always clinically significant [2]. Gastrointestinal disease and malignancy are the most common predisposing factors for clinically significant bacteremia [2]. Although three cases of inflammatory bowel disease with Eggerthella strains in feces have been reported in the literature, Eggerthella bacteremia in inflammatory bowel disease patients has never been reported [1]. Here we report the case of a young African-American female with Crohn's disease and Eggerthella lenta bacteremia.

\section{Case Report}

A 21-year-old African-American female presented to our hospital with complaints of abdominal pain, nausea, vomiting and diarrhea. Her symptoms initially began approximately 2 weeks before admission with on and off, cramping lower abdomen pain associated with loose, nonbloody diarrhea. Her emesis was nonbloody and nonbilious, associated with severe nausea. She was initially admitted to our hospital for 2 days and discharged home after resolved partial small bowel obstruction approximately 3 days prior to present presentation. The patient denied any fevers, chills, eating food outside, sick contacts, recent antibiotic use, previous abdominal surgeries or any similar symptoms in the past. The patient looked very weak and toxic. Her vital signs were of significance since she had a temperature of $36.6^{\circ} \mathrm{C}$, a pulse rate of $128 \mathrm{bpm}$, blood pressure of $110 / 70 \mathrm{mmHg}$ and a respiratory rate of 18 breaths/min. Abdominal examination showed diffuse tenderness with guarding and absent bowel sounds. Admission white count was $12.4 \mathrm{k} / \mathrm{mm}^{3}$ with no bands, hemoglobin $11.2 \mathrm{~g} / \mathrm{dl}$, platelets $403 \mathrm{k} / \mathrm{mm}^{3}$, anion gap of 16, otherwise normal metabolic panel, urinalysis, liver function tests, amylase, lipase, negative Clostridium difficile PCR and negative gonococcal/chlamydiae PCR. Computed tomography abdomen and pelvis with contrast showed findings consistent with severe distal small bowel obstruction with a transition point in the distal ileum and an associated $8.5 \mathrm{~cm}$ ileal dilatation proximal to transition point (FIGURe 1). The patient was taken to the operating room for urgent exploratory laparatomy and had ileocecal resection with primary ileocolic anastomosis. Grossly resected ileal specimen appeared inflamed with strictures. Abdominal fluid was sent for cultures and resected ileal specimen was sent for pathological evaluation. Her postoperative course was complicated by sepsis with fever spikes, tachycardia, hypotension and had to be monitored in the intensive care unit. Blood cultures, urine cultures and abdominal fluid cultures were sent and she was started on intravenous vancomycin and piperacillin/tazobactam. Her blood pressure improved with intravenous hydration and was transferred to the general medical floor after 3 days. Pathology specimen showed mucosal ulceration with inflammation, crypt abscesses and nodular lymphoid aggregates consistent with Crohn's disease. Despite being on

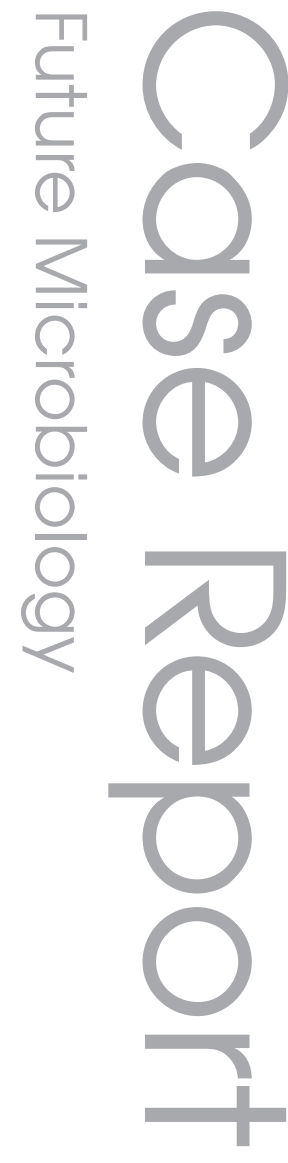

\section{Keywords}

- bacteremia $=$ Crohn's

disease = Eggerthella lenta

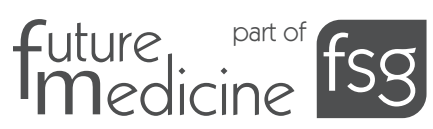




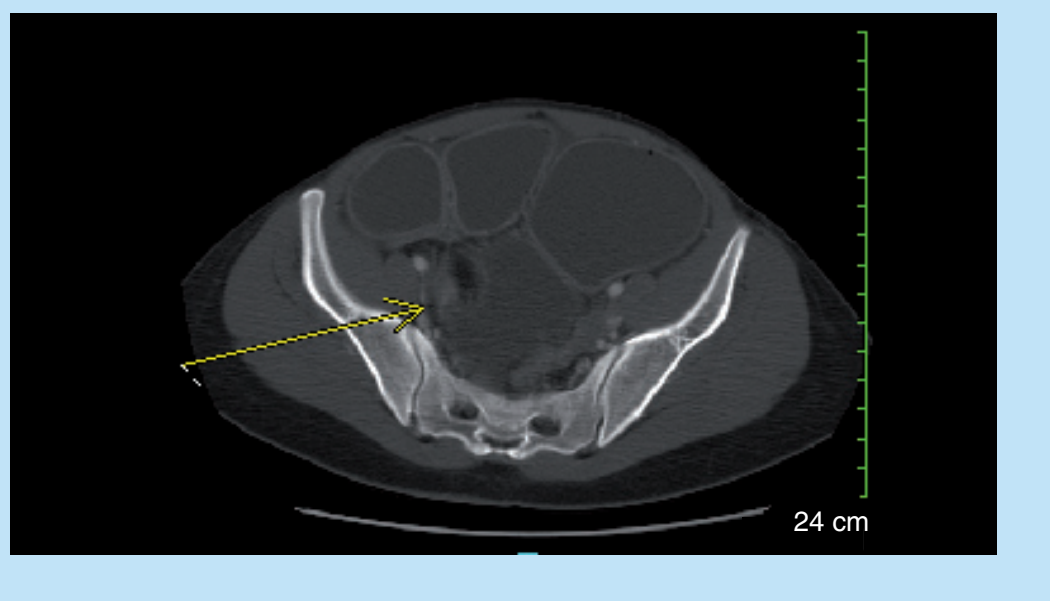

Figure 1. Computed tomography abdomen/pelvis with intravenous contrast showing severe distal small bowel obstruction with a transition point in the distal ileum. The scale bar is equal to $24 \mathrm{~cm}$.

antibiotics, patient continued to spike fevers over $39.4^{\circ} \mathrm{C}$ for approximately 7 days. An infectious disease service was consulted. Abdominal fluid cultures, initial blood cultures, urine cultures and $C$. difficile PCR were negative. Repeat computed tomography abdomen and pelvis showed findings consistent with postoperative ileus with no evidence of any abscesses or anastomosis leak. Although blood cultures on admission were negative, she was bacteremic in four sets of blood cultures drawn postoperatively on days 1 and 2, positive for Grampositive rods from anaerobic bottles only. The piperacillin/tazobactam was discontinued and meropenem and metronidazole were added to vancomycin for broader coverage. A repeat exploratory laparatomy was performed given her fever spikes and nonresolving ileus on day 10 post-operation. There was no evidence of abscess or active infection but the anastomosis site was found to be disrupted. She underwent focal re-resection with reanastomosis. After the second surgery patient's ileus slowly started to resolve. On postoperative day 10, the blood cultures were identified as Eggerthella lenta (Alverno clinical laboratory, IN, USA, by VITEK $^{\circledR} 2$ Anaerobic card). The infectious diseases service discontinued intravenous vancomycin and metronidazole, and she was continued on meropenem for 4 more days. Her fever and leucocytosis slowly resolved. Following resolution of her symptoms she was discharged home on postoperative day 17 with plans for outpatient esophagogastroduodenoscopy and colonoscopy for evaluation of the extent of Crohn's disease.

\section{Discussion}

Eggerthella lenta is an anaerobic, nonsporeforming Gram-positive rod that belongs to the family Coriobacteriaceae. It was first reported in 1935 by Arnold Eggerth and named after him. These bacteria occur singly or in short chains as commensals in human intestinal flora. It was previously classified as Eubacterium lentum until 1999, after which it was reclassified as Eggerthella based on 16S rRNA sequencing [1]. Its identification is often difficult owing to its fastidious and slow-growing nature, as was evident in our patient. Bacteremia is thought to occur secondary to translocation into blood from the gut. Predisposing diseases include GI tract disease, malignancy, hepatobiliary disease, immobilization, bed sores, diabetes mellitus and stroke. Infections of the gastrointestinal and genital tracts by Eggerthella have also been reported [2].

Except for Propionibacterium acnes, bacteremia from anaerobic, Gram-positive bacilli should always be managed cautiously. Although these are common gut commensals, their bacteremia is associated with high mortality, especially in immunocompromised patients [2] Prompt initiation of antibiotic treatment and identification of source should be sought following speciation of the isolates. Eggerthella species are usually sensitive to penicillins and metronidazole with variable sensitivity to vancomycin [3].

Although Eggerthella bacteremia is becoming more common than expected with significant morbidity and mortality $[4,5]$, we report its first association with Crohn's disease. Theoretically it is logical for Eggerthella translocation from gut to blood given the wall inflammation, it is not known whether this occurs with greater frequency or contributes to excess morbidity in Crohn's disease. Eggerthella isolation from blood cultures in a previously healthy patient should prompt looking for any underlying diseases of the GI tract and malignancy.

\footnotetext{
Financial \& competing interests disclosure

The authors have no relevant affliations or financial involvement with any organization or entity with a financial interest in or financial conflict with the subject matter or materials discussed in the manuscript. This includes employment, consultancies, honoraria, stock ownership or options, expert testimony, grants or patents received or pending, or royalties.

No writing assistance was utilized in the production of this manuscript.
} 


\section{Bibliography}

1. Kageyama A, Benno Y, Nakase T: Phylogenetic evidence for the transfer of Eubacterium lentum to the genus Eggerthella as Eggerthella lenta gen. nov., comb. nov. Int. J. Syst. Bacteriol. 49(Pt 4), 1725-1732 (1999).

2. Lau SK, Woo PC, Fung AM, Chan KM, Woo GK, Yuen KY: Anaerobic, nonsporulating, Gram-positive bacilli bacteraemia characterized by $16 \mathrm{~S}$ rRNA gene sequencing. J. Med. Microbiol. 53(Pt 12), 1247-1253 (2004).

3. Bok CW, Ng YS: Eggerthella lenta as a cause of anaerobic spondylodiscitis. Singapore Med. J. 50(12), E393-E396 (2009).

4. Chan RC, Mercer J: First Australian description of Eggerthella lenta bacteraemia identified by $16 \mathrm{~S}$ rRNA gene sequencing. Pathology 40(4), 409-410 (2008).
5. Lau SK, Woo PC, Woo GK et al.: Eggerthella hongkongensis sp. nov. and Eggerthella sinensis sp. nov., two novel Eggerthella species, account for half of the cases of Eggerthella bacteremia. Diagn. Microbiol. Infect. Dis. 49(4), 255-263 (2004). 